\title{
Diversity, equity and inclusion: we are in it for the long run
}

\author{
We are launching a series on Diversity, Equity and Inclusion in medical research and are redoubling our \\ commitment to representation in our pages.
}

T his issue of Nature Medicine marks the launch of a Series on Diversity, Equity and Inclusion (DEI) in medical research. Every month we will be bringing to our readers a spectrum of viewpoints from critical stakeholders who are shaping the DEI debate.

We start off the series with a problem that concerns every researcher worldwide: funding. Using the United States as case study, Folakemi T. Odedina and Mariana C. Stern present an overview of challenges that scientists from under-represented minority groups, such as researchers who are Black, Latinx or Indigenous, face in the context of securing research funding. Awareness of this problem has increased in the past 2 years, in the awakening of a broader acknowledgment of the impact of structural racism on every layer of society. But the authors point out that disparities in medical research are a consequence of long-term factors, including the paucity of research funding for the study of health disparities, a biased scientific review process and, most recently, the increasing burden of diversity committees and taskforces. The solution, they say, begins with remedying biases in the funding application process and prioritizing research that addresses health disparities.

In the United States, the Biden administration's response to structural racism and under-representation in biomedical research has coalesced into the National Institutes of Health (NIH) UNITE initiative. Marie A. Bernard, Chief Officer for Scientific Workforce Diversity at the NIH, and her colleagues discuss the launch and priorities of the initiative, as well as how the NIH intends to measure the mid- and long-term success of the program. In their words, the NIH is committed to increasing the participation of under-represented groups in science and medicine, through changes in funding and recruitment models, as well as transparency about the workforce and reviewer demographics at funders, institutions and publishers. The intention is that these actions will also provide a blueprint for the global research community.
Any discussion on the role of funders in increasing DEI in medical research must involve non-governmental stakeholders as well. The contribution of philanthropic grant-making to the overall funding landscape has grown substantially over the past 25 years. In 2019, the US National Science Foundation estimated that up to $44 \%$ of all basic science research expenditures at American universities and research institutes was directly or indirectly funded through philanthropy. It is critical that philanthropic funders review their own practices and processes and that they are transparent in their approach to addressing gaps in representation in their own funding portfolios. Cori Bargmann, head of science at the Chan Zuckerberg Initiative, and her colleagues Anne Claiborne and Hannah Valantine discuss one such philanthropic approach toward DEI in medical research. In their view, racial equity and global inclusion are essential if biomedical science is to fulfill its mission to improve human health.

Addressing disparities in the funding of medical research also must encompass the creation of opportunities that generate a diverse workforce and foster a culture of inclusivity in science. Marcia McNutt, president of the US National Academy of Sciences, and Laura Castillo-Page, Chief Diversity and Inclusion Officer for the National Academies of Science, Engineering, and Medicine, discuss the academies' recent efforts to diversify its own ranks - which, in their perspective, for too many decades saw the appointment of new members who were mostly white men. Representation at the leadership level has a critical impact on the advancement of inclusivity in STEMM fields, through role modeling, embracing of diversity and creating a research culture that cares about and values differences.

The viewpoints presented in this month's issue are intended to serve as a starting point for a much broader discussion about diversity, inclusion and equity in medical research. In upcoming months, we will be tackling issues such as the lack of diversity in medical research data, its consequences and how to close that gap, research priorities in under-represented groups and unfair practices in collaborative research, as well as what the challenges of building a diverse scientific workforce look like in other areas of the globe. We invite our readers to contribute content across all sections of the journal, including research papers focused on interventions to reduce health inequities.

Finally, we want to report back on our own progress toward diversity and inclusion in our pages. We are focusing our efforts on increasing the diversity of peer reviewers and authorship of contributed content for the front end of the journal. These are two areas in which we can actively engage with a more diverse universe of contributors and ensure that our peer reviewers and invited authors do reflect the entire spectrum of the journal's global readership.

For the past 2 years, we have been asking all our peer reviewers, after completion of a review, to provide information on their geographical location, self-identified gender and career stage. We will be sharing the aggregated results on an annual basis starting with 2021, with publication in the subsequent year. This effort, together with peer-reviewer recognition - in which reviewers can choose to be named as referees of the studies we publish - will help us to offer more transparency about what our referee pool looks like. Reviewers are critical partners in our decisions of what to publish in Nature Medicine. Striving for diversity of research in our pages necessarily requires working with a far more diverse range of contributors that we currently have. We pledge to do our part, but you can also help us by naming colleagues of diverse backgrounds and career stages when we invite you to participate in peer review but you are not available to review a paper yourself.

Perhaps the most challenging aspect of striving for diversity, equity and inclusion is the realization that there is no instant solution. Progress will require patience, persistence, continuous commitment and self-assessment. We are in it for the long run.

Published online: 11 November 2021 https://doi.org/10.1038/s41591-021-01582-5 